\title{
CORROSÃO ALCALINA DO ALUMÍNIO PARA PRODUÇÃO DE HIDROGÊNIO CONSIDERANDO DIFERENTES GEOMETRIAS, TEMPERATURAS E CONCENTRAÇÕES
}

\author{
L.C.C.B.OLIVEIRA, R.M.PIGNATA e S.C. DANTAS ${ }^{1}$ \\ ${ }^{1}$ Universidade Federal do Triângulo Mineiro, Departamento de Engenharia Química \\ E-mail para contato: sandra@icte.uftm.edu.br \\ bonfim lorrane@hotmail.com
}

\begin{abstract}
RESUMO - Diante do cenário enfrentado de mudanças climáticas e falta de recursos energéticos, a produção de hidrogênio surge como uma eficiente fonte alternativa de energia renovável. Este estudo visa a produção de hidrogênio por meio da corrosão alcalina de latinhas de alumínio, através da análise da influência de alguns fatores, tais como a temperatura, concentração de catalisador e geometria do alumínio, sobre a reação. Adotaram-se as temperaturas de $25^{\circ} \mathrm{C}$, $35^{\circ} \mathrm{C}$ e $45^{\circ} \mathrm{C}$, forma do alumínio em tiras, placas e quadrados e a concentração do hidróxido de sódio variou em $1 \mathrm{M}, 2 \mathrm{M}$ e $3 \mathrm{M}$. Os experimentos mostraram que a forma em tiras obteve a maior produção de hidrogênio em relação às outras geometrias. Independente da concentração de $\mathrm{NaOH}$ e da geometria, o aumento de temperatura implicou em uma maior produção de $\mathrm{H}_{2}$. Já em relação ao catalisador, o maior rendimento de hidrogênio foi observado ao empregar a concentração de $\mathrm{NaOH} 2 \mathrm{M}$.
\end{abstract}

\section{INTRODUÇÃO}

A sociedade industrializada moderna necessita de fontes de energia para que se desenvolva. Atualmente, estas principais fontes energéticas advêm dos combustíveis fósseis (Porciúncula, 2013). No entanto, as preocupações acerca de questões ambientais, como a escassez de energia, esgotamento de recursos, e do aquecimento global, exigem o desenvolvimento de energias renováveis ao invés dos combustíveis fósseis (Kumagai et al., 2014). A produção de energia a partir do hidrogênio pode ser considerada como uma alternativa energética promissora, a qual ainda não é muito usual. No entanto, têm atraído muita atenção de pesquisadores, pelo principal fator de que sua utilização não emite gases poluentes (Chai et al., 2014).

Como destacado por Sharma e Ghoshal (2015) existem diferentes maneiras de se produzir o hidrogênio, as quais podem ser originada de meios renováveis ou não. Alguns métodos se destacam como as reações de reforma, principalmente em relação ao metano ou etanol, eletrólise da água, decomposição por arco de plasma, termólise da água e conversão termoquímica da biomassa.

Outro método de geração de hidrogênio, que se mostra muito eficiente, é por meio da corrosão alcalina de metais. E como revelado por Ilyukhina et al. (2012), o material mais apropriado para isso é o alumínio devido a algumas propriedade apresentadas, tais como 
elevada eficiência na produção de hidrogênio $\left(1,241 . \mathrm{g}^{-1}\right)$, segurança ambiental dos produtos de reação, segurança de armazenamento e transporte e baixo preço. Contudo, para que esta reação aconteça é necessário a adição de um catalisador, como por exemplo, uma base forte. Somente deste modo a película de óxido de alumínio formada pode ser degradada e assim ocorrer a produção de hidrogênio, como mostrado por Teng et al. (2012) e Chai et al. (2014), e representado pelas equações I, II e III.

$$
\begin{aligned}
& 2 \mathrm{Al}+6 \mathrm{H}_{2} \mathrm{O} \rightarrow 2 \mathrm{Al}(\mathrm{OH})_{3}+3 \mathrm{H}_{2} \\
& 2 \mathrm{Al}+6 \mathrm{H}_{2} \mathrm{O}+2 \mathrm{NaOH} \rightarrow 2 \mathrm{NaAl}(\mathrm{OH})_{4}+3 \mathrm{H}_{2} \\
& 2 \mathrm{NaAl}(\mathrm{OH})_{4} \rightarrow 2 \mathrm{NaOH}+2 \mathrm{Al}(\mathrm{OH})_{3}
\end{aligned}
$$

A produção de hidrogênio a partir do alumínio, além das vantagens citadas anteriormente, destaca-se pela alternativa de utilização de refugos a base de alumínio, como latas de bebidas, peças de dispositivos eletrônicos e brinquedos, na geração de energia, o que incentivaria ainda mais a reciclagem deste tipo de material (Porciúncula, 2013). De acordo com Macanás et al. (2011), o alumínio é o segundo metal mais utilizado no mundo, e este método está intimamente relacionado à reciclagem do alumínio. Desta forma, o objetivo deste trabalho é a produção de hidrogênio por meio da corrosão alcalina de latas de alumínio, assim como a verificação da influência da concentração de $\mathrm{NaOH}$, da geometria do alumínio e da temperatura de reação utilizada.

\section{PROCEDIMENTO EXPERIMENTAL}

Para a realização do experimento, algumas latas de alumínio foram arrecadadas, em seguida cortadas e lixadas, até que o revestimento externo e interno fosse removido. Consideraram-se três tipos de geometria para o corte das latinhas: em tiras, quadrados e placas, as quais podem ser vistas na Figura 1.A.

Figura 1 - (A) Imagem das diferentes geometrias e (B) representação do sistema reacional empregado.

(A)

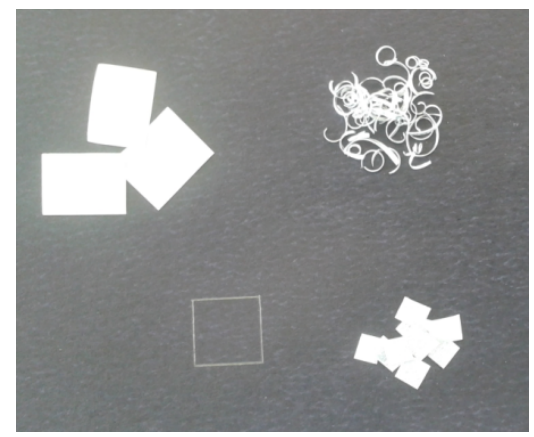

(B)

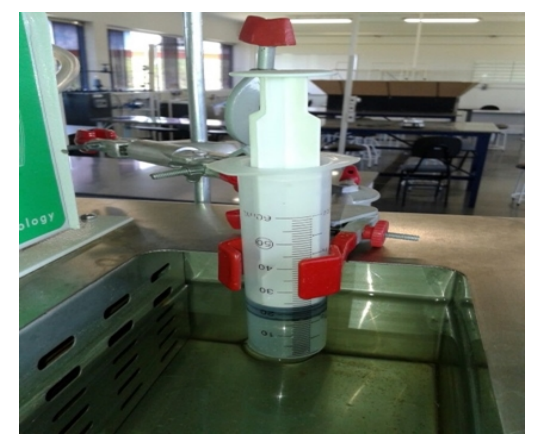

Para os experimentos foram avaliados três diferentes temperaturas $\left(25^{\circ} \mathrm{C}, 35^{\circ} \mathrm{C}\right.$ e $\left.45^{\circ} \mathrm{C}\right)$ e três concentrações de $\mathrm{NaOH}(1 \mathrm{M}, 2 \mathrm{M}$ e $3 \mathrm{M})$. Os ensaios foram avaliados utilizando uma 
massa de $0,030 \mathrm{~g}$ de lata de alumínio, submetida às diferentes temperaturas e concentrações de hidróxido de sódio, obtendo se desta forma um total de 27 ensaios.

O sistema empregado para verificar a produção de hidrogênio foi simples, composto por um banho termostático e um suporte para uma seringa de $60 \mathrm{ml}$. Vale ressaltar que a seringa foi vedada com tampão de plástico, não apresentando qualquer risco de vazamento. Conforme o hidrogênio era produzido, o êmbolo da seringa se deslocava, indicando o volume de hidrogênio produzido em função do tempo. A representação do sistema descrito pode ser visto pela Figura 1.B.

\section{RESULTADOS E DISCUSSÕES}

Os resultados obtidos através dos experimentos revelam a dependência da reação alumínio-água com a geometria, temperatura e concentração de solução alcalina, e estão representados pelas Figuras 2, 3 e 4. Analisando a Figura 2, para a geometria de placas, notase uma maior produção de hidrogênio com o aumento de temperatura e da concentração de $\mathrm{NaOH}$.

Figura 2 - Volume de hidrogênio produzido para as placas de alumínio nas temperaturas de $25^{\circ} \mathrm{C}(\mathrm{A}), 35^{\circ} \mathrm{C}(\mathrm{B})$ e $45^{\circ} \mathrm{C}(\mathrm{C})$. E rendimento de hidrogênio na temperatura de $45^{\circ} \mathrm{C}(\mathrm{D})$, onde $\mathbf{\square}, \bullet$ e $\Delta$ representam respectivamente, as concentrações de1M, $2 \mathrm{M}$ e $3 \mathrm{M}$.



(A)

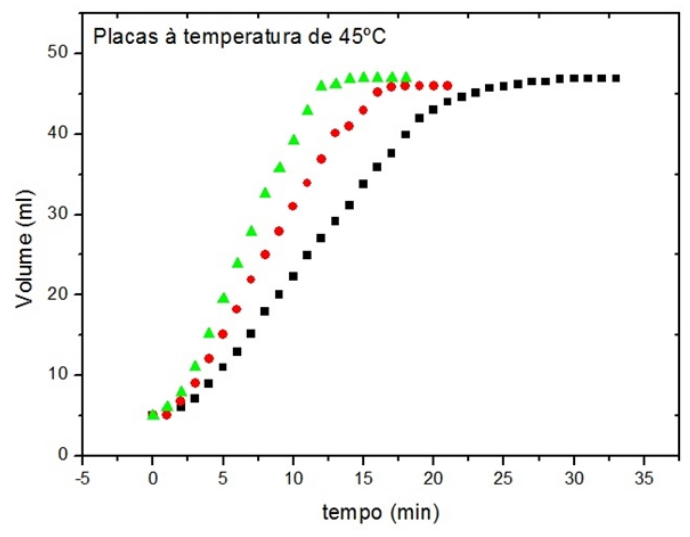



(B)

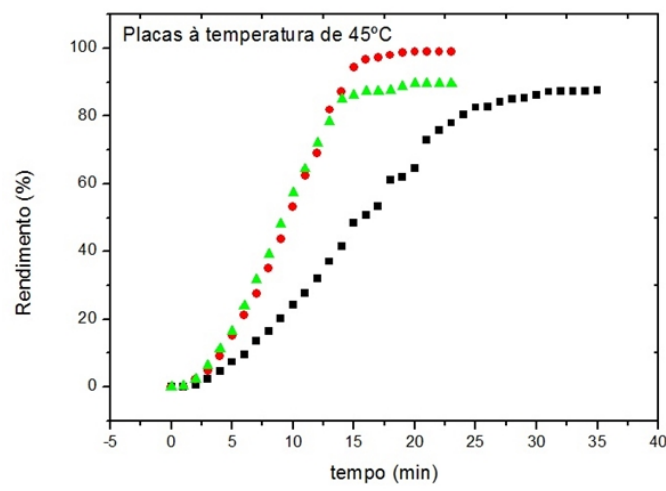

(D) 
No entanto, vale ressaltar que não houve muitas diferenças entre as maiores concentrações as quais apresentaram muitos pontos em comum. Analisando o rendimento (Figura 2.D) para a placa à temperatura de $45^{\circ} \mathrm{C}$, observa-se que o maior rendimento é alcançado ao se empregar a concentração de $2 \mathrm{M}$, cerca de um pouco mais de $9 \%$ em relação a concentração de $3 \mathrm{M}$. A diferença observada entre o volume de hidrogênio formado (Figura 2.C) e o rendimento para a produção de hidrogênio está relacionada a massa utilizada em cada experimento. Como para esta geometria utilizou-se uma única placa de alumínio, uma pequena mudança na massa resultou em alteração significativa com relação ao rendimento.

Observando a Figura 3, para a geometria de quadrados, nota-se que o aumento de temperatura resultou em uma produção de hidrogênio maior e mais rápida, assim como para a geometria em placas. Além disso, a concentração de $2 \mathrm{M}$ também se destaca pela maior produção de hidrogênio quando comparada com as outras concentrações utilizadas e maior rendimento (Figura 3.D).

Figura 3 - Volume de hidrogênio produzido para a geometria de quadrados nas temperaturas de $25^{\circ} \mathrm{C}(\mathrm{A}), 35^{\circ} \mathrm{C}(\mathrm{B})$ e $45^{\circ} \mathrm{C}(\mathrm{C})$. E rendimento de hidrogênio na temperatura de $45^{\circ} \mathrm{C}$ (D), onde $\square, \bullet$ e $\Delta$ representam respectivamente, as concentrações de1M, $2 \mathrm{M}$ e $3 \mathrm{M}$.
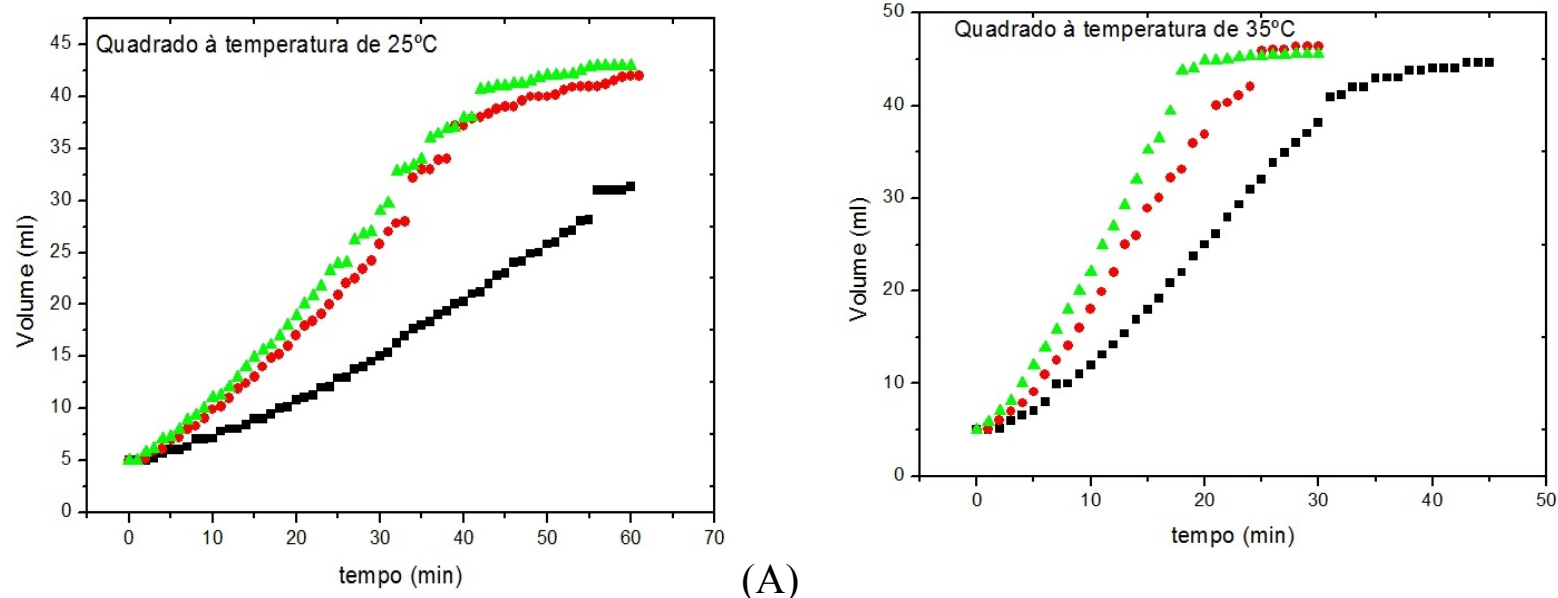

(A)


(D) 
A Figura 4 apresenta a quantidade de hidrogênio formada para a geometria de tiras. Observa-se que, assim como para as outras geometrias, o aumento de temperatura resultou na maior produção de hidrogênio. Em relação à concentração, nota-se que o aumento da concentração molar da solução alcalina resulta em maiores volumes de hidrogênio produzido. Analisando o rendimento (Figura 4.D) para a forma em tiras à temperatura de $45^{\circ} \mathrm{C}$, observase que o maior rendimento é para a concentração de $3 \mathrm{M}$, mesmo que seja mínima a diferença se comparado com a concentração de $2 \mathrm{M}$, menos de $3 \%$.

Figura 4 - Volume de hidrogênio produzido para a geometria de tiras nas temperaturas de $25^{\circ} \mathrm{C}(\mathrm{A}), 35^{\circ} \mathrm{C}(\mathrm{B})$ e $45^{\circ} \mathrm{C}(\mathrm{C})$. E rendimento de hidrogênio na temperatura de $45^{\circ} \mathrm{C}$ (D), onde $\mathbf{\square}, \bullet$ e $\triangle$ representam respectivamente, as concentrações de1M, $2 \mathrm{M}$ e $3 \mathrm{M}$
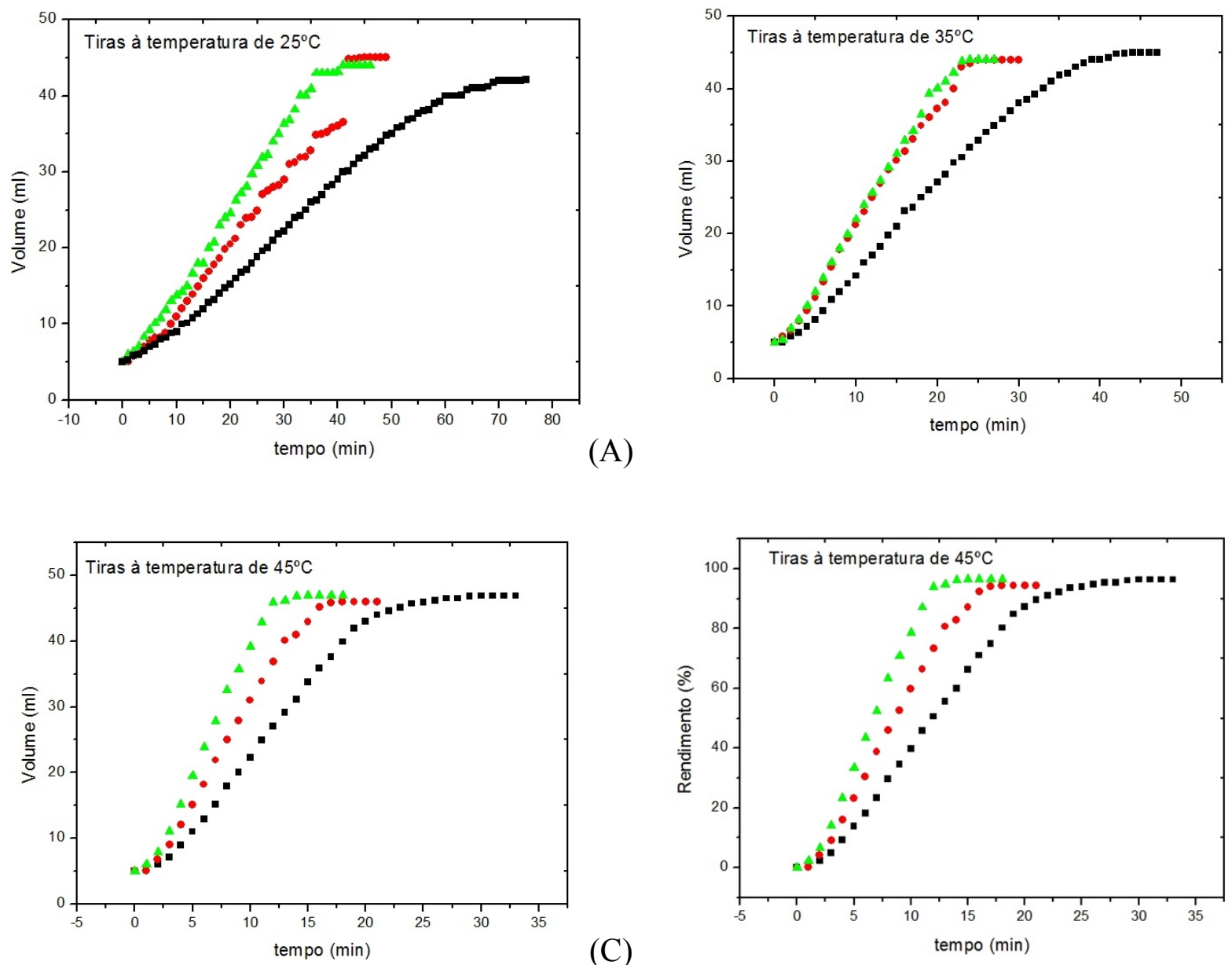

(D)

Comparando as diferentes geometrias, nota-se que a forma em tiras, como esperado, apresentou a mais rápida produção de hidrogênio, e isso ocorreu devido a maior área de contato existente para que a reação acontecesse. Além disso, não foi observada muita diferença na produção entre as concentrações de $2 \mathrm{M}$ e $3 \mathrm{M}$, mesmo sendo a produção de hidrogênio mais acelerada a $3 \mathrm{M}$, em muitos resultados a maior produção alcançada era quando se empregava a concentração de $2 \mathrm{M}$, e isso aconteceu de maneira geral, entre todas as formas e temperatura. Assim como o aumento de temperatura implicou em uma maior produção de hidrogênio. Já em relação ao rendimento analisado, considerando à temperatura 
fixa à $45^{\circ} \mathrm{C}$, a diferença obtida entre as maiores concentrações foi pequena. No entanto, a diferença entre elas e a de $1 \mathrm{M}$ foi bastante significativa.

\section{CONCLUSÕES}

Diante do que foi exposto, a corrosão alcalina de alumínio se mostra como uma alternativa relevante na produção de hidrogênio. Muitos são os motivos para se investir neste tipo de procedimento, seja visando o desenvolvimento de novas células combustíveis ou mesmo diminuindo a formação de dióxido de carbono, que é um dos gases responsáveis pelo aquecimento do planeta. Além disso, este método está intimamente relacionado à reciclagem do alumínio, que é considerado o segundo metal mais utilizado no mundo. Os ensaios revelam que, de maneira geral para todas as geometrias avaliadas, uma temperatura mais elevada e concentração de $2 \mathrm{M}$ resultou em uma maior produção de hidrogênio. Sendo que, dentre estas geometrias, a que mais se destacou pela maior formação de hidrogênio em menor tempo foi a forma em tiras. Entretanto, apesar dos valores próximos, avaliando o rendimento, o quadrado foi a geometria que mais se destacou na temperatura de $45^{\circ} \mathrm{C}$. Outro importante ponto a se destacar é em relação à solução empregada a qual é fundamental para que haja a degradação dos óxidos formados e assim a produção do hidrogênio.

\section{REFERÊNCIAS}

CHAI, Y.J.; MENG, H.X.; JIA, Y.Y.; SHEN, J.; HUANG, Y.M.; WANG, N. Hydrogen generation by aluminum corrosion in cobalt (II) chloride and nickel (II) chloride aqueous solution. Energy, v. 68, p. 204 - 209, 2014.

ILYUKHINA, A.V.; ILYUKHIN, A.S.; SHKOLNIKOV, E.I. Hydrogen generation from water by means of activated aluminum. International Journal of Hydrogen Energy, v.37 p.6382-6387, 2012.

KUMAGAI, S.; ALVAREZ, J.; BLANCO, P.H.; WU, C.; YOSHIOKA, T.; OLAZAR, M.; WILLIAMS, P.T. Novel Ni-Mg-Ca catalyst for enhanced hydrogen production for the pyrolysis-gasification of a biomass/plastic mixture. Journal of Analytical and Applied Pyrolysis, In Press, 2014.

MACANÁS, J.; SOLER, L.; CANDELA, A.M.; MUÑOZ, M.; CASADO, J. Hydrogen generation by aluminum corrosion in aqueous alkaline solutions of inorganic promoters: The AlHidrox process. Energy, v. 36, p. 2493-2501, 2011.

PORCIÚNCULA, C.B; Simulação e operação de célula de combustível com geração in situ de hidrogênio através da corrosão alcalina do alumínio. Tese de doutorado, DEQ/UFRS, 2013.

SHARMA, S.; GHOSHAL, S.K.; Hydrogen the future transportation fuel: From production to applications. Renewable and Sustainable Energy Reviews, v.43, p. 1151-1158, 2014.

TENG, H.; LEE, T.; CHEN, Y.; WANG, H.; CAO, G. Effect of $\mathrm{Al}(\mathrm{OH})_{3}$ on the hydrogen generation of aluminum-water system. Journal of Power Sources, v. 219 p.16-21, 2012. 